\title{
CHEETAH: \\ A Next Generation Thermochemical Code
}

\author{
L. Fried \\ P. Souers
}

November 1994

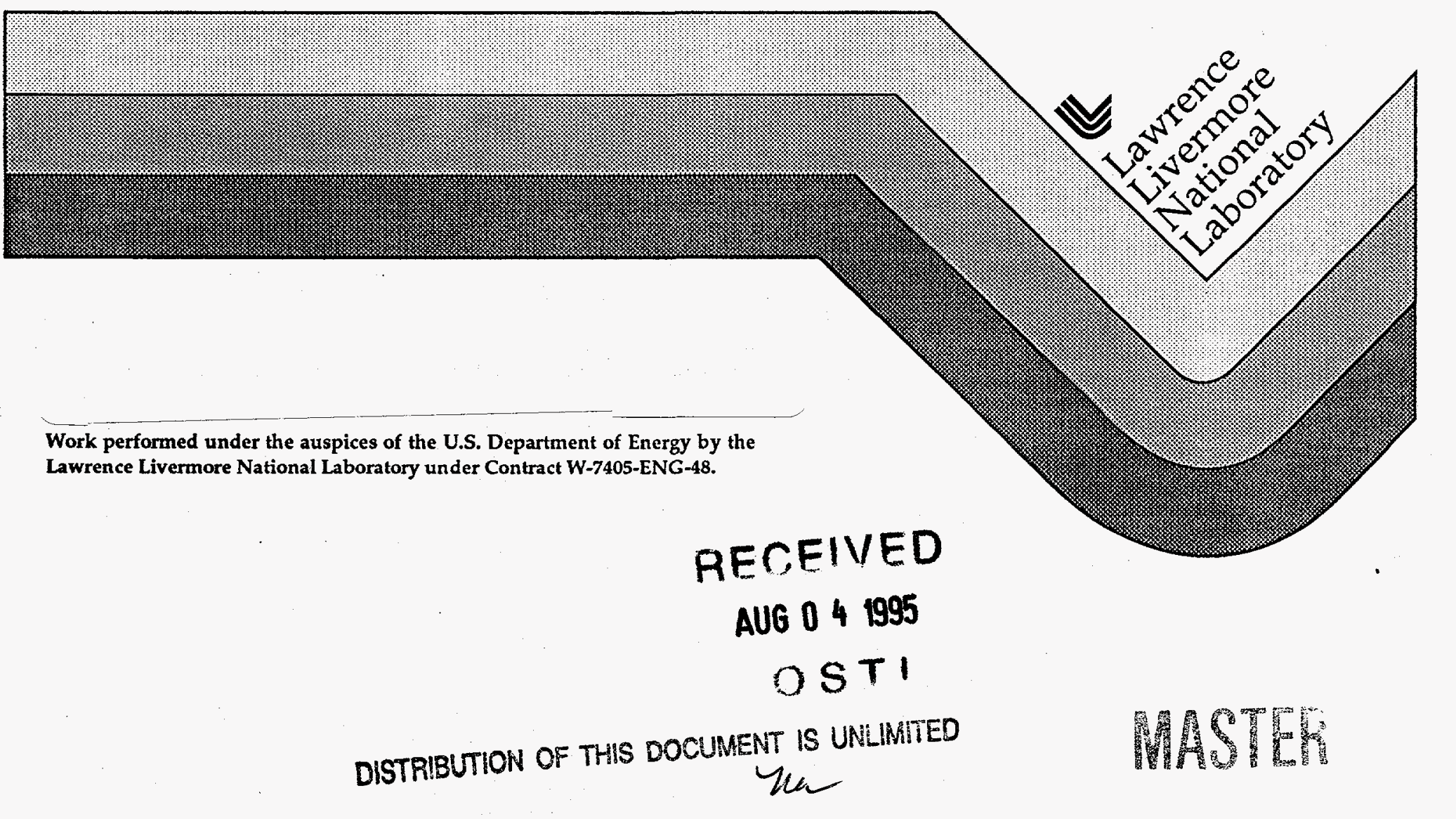




\section{DISCLAIMER}

This document was prepared as an account of work sponsored by an agency of the United States Government. Neither the United States Government nor the University of California nor any of their employees, makes any warranty, express or implied, or assumes any legal liability or responsibility for the accuracy, completeness, or usefulness of any information, apparatus, product, or process disclosed, or represents that its use would not infringe privately owned rights. Reference herein to any specific commercial product, process, or service by trade name, trademark, manufacturer, or otherwise, does not necessarily constitute or imply its endorsement, recommendation, or favoring by the United States Government or the University of California. The views and opinions of authors expressed herein do not necessarily state or reflect those of the United States Government or the University of California, and shall not be used for advertising or product endorsement purposes.

This report has been reproduced directly from the best available copy.

Available to DOE and DOE contractors from the Office of Scientific and Technical Information P.O. Box 62, Oak Ridge, TN 37831

Prices available from (615) 576-8401, FTS 626-8401

Available to the public from the National Technical Information Service

U.S. Department of Commerce 5285 Port Royal Rd. Springfield, VA 22161

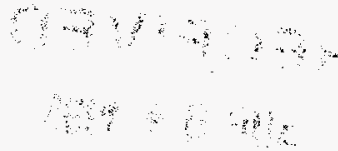




\section{DISCLAIMER}

Portions of this document may be illegible in electronic image products. Images are produced from the best available original document. 


\title{
CHEETAH: A next generation thermochemical code
}

\author{
Laurence E. Fried and P. Clark Souers
}

(510) $422-7796$

\begin{abstract}
CHEETAH[1] is an effort to bring the TIGER thermochemical code[2] into the 1990s. A wide variety of improvements have been made in Version 1.0. We have improved the robustness and ease of use of TIGER. All of TIGER's solvers have been replaced by new algorithms. We find that CHEETAH solves a wider variety of problems with no user intervention (e.g. no guesses for the C-J state) than TIGER did. CHEETAH has been made simpler to use than TIGER; typical use of the code occurs with the new standard run command. CHEETAH will make the use of thermochemical codes more attractive to practical explosive formulators.

We have also made an extensive effort to improve over the results of TIGER. CHEETAH's version of the $B K W$ equation of state (BKWC) is able to accurately reproduce energies from cylinder tests; something that other BKW parameter sets have been unable to do. Calculations performed with BKWC execute very quickly; typical run times are under 10 seconds on a workstation.

In the future we plan to improve the underlying science in CHEETAH. More accurate equations of state will be used in the gas and the condensed phase. A kinetics capability will be added to the code that will predict reaction zone thickness. Further ease of use features will eventually be added; an automatic formulator that adjusts concentrations to match desired properties is planned.
\end{abstract}

\section{Overview}

\subsection{What CHEETAH does}

Below we give a non-technical explanation of what CHEETAH does. CHEETAH is a thermochemical code. It solves thermodynamic equations between product species to find chemical equilibrium. For instance, in a system comprised solely of condensed carbon, gaseous $\mathrm{CO}$, and gaseous $\mathrm{CO}_{2}$, CHEETAH can find the equilibrium of the reaction

$$
\mathrm{C}+\mathrm{CO}_{2} \leftrightarrow 2 \mathrm{CO}
$$

at a specified pressure and temperature. C-J theory says that the detonation point is a state in thermodynamic and chemical equilibrium, so CHEETAH can predict the properties of this state. 
From these properties and elementary detonation theory come the detonation velocity and other performance indicators. CHEETAH can calculate thermodynamic states where the pressure and temperature are not explicitly indicated. For instance, we can specify the volume and the entropy instead of the pressure and temperature. These types of calculations implicitly define pressure and temperature, i.e.

$$
\begin{aligned}
& V(P, T)=V_{0} \\
& S(P, T)=S_{0}
\end{aligned}
$$

$(\mathrm{V}, \mathrm{S})$ points play an important role when undertaking an adiabatic expansion from the C-J state.

Thermodynamic equilibrium can be found by balancing chemical potentials. The above carbon example leads to the equation

$$
\mu_{\mathrm{C}}+\mu_{\mathrm{CO}_{2}}=2 \mu_{\mathrm{CO}}
$$

The chemical potentials of condensed species are just functions of pressure and temperature, while the potentials of gaseous species depend on concentrations:

$$
\begin{aligned}
\mu_{\mathrm{C}} & =\mu_{\mathrm{C}}(P, T) \\
\mu_{\mathrm{CO}_{2}} & =\mu_{\mathrm{CO}_{2}}\left(P, T, N_{\mathrm{CO}_{2}}, N_{\mathrm{CO}}\right) \\
\mu_{\mathrm{CO}} & =\mu_{\mathrm{CO}}\left(P, T, N_{\mathrm{CO}_{2}}, N_{\mathrm{CO}}\right)
\end{aligned}
$$

Here, $\mathrm{N}_{\mathrm{CO}_{2}}$ is the number of molecules of $\mathrm{CO}_{2}$. Recall that the chemical potential is the Gibbs free energy difference obtained from adding one more molecule into the system:

$$
\mu_{\mathrm{C}}=G\left(P, T, N_{\mathrm{CO}_{2}}, N_{\mathrm{C}}+1 . N_{\mathrm{CO}}\right)-G\left(P, T, N_{\mathrm{CO}_{2}}, N_{\mathrm{C}}, N_{\mathrm{CO}}\right)
$$

Balancing the chemical potentials is the same as minimizing $G$. In fact many thermochemical codes (notably CHEQ[3]) numerically minimize $G$ instead of bothering with chemical potentials.

There are no chemical reactions explicitly specified in CHEETAH. How then, you might ask, does CHEETAH get the chemical reactions for balancing chemical potentials? The answer is that the reactions used don't matter, as long as there are enough of them to change concentrations arbitrarily; the answer will come out the same no matter what reactions are used. CHEETAH manufactures a decomposition reaction for each product molecule. This reaction is an internal construct of the code and does not have to occur physically. The product molecule (called a "constituent" in the language of TIGER) decomposes into a small number of "component" molecules. The number of components is equal to the number of elements in the problem. For example CHEETAH might choose $\mathrm{C}$ and $\mathrm{CO}$ as components. The decomposition reactions would then be:

$$
\begin{aligned}
\mathrm{C} & \leftrightarrow \mathrm{C} \\
\mathrm{CO} & \leftrightarrow \mathrm{CO} \\
\mathrm{CO}_{2} & \rightarrow 2 \mathrm{CO}-\mathrm{C}
\end{aligned}
$$

Since decomposition reactions in CHEETAH aren't real, they can have negative stoichiometric coefficients. The first two reactions are trivial and therefore have no effect on the chemical equilibrium. 
The equations solved by CHEETAH are

$$
\begin{aligned}
\mu_{\mathrm{CO}_{2}}\left(P, T, x_{\mathrm{CO}}, x_{\mathrm{CO}_{2}}\right) & =2 \mu_{\mathrm{CO}}\left(P, T, x_{r m C O}, x_{\mathrm{CO}_{2}}\right)-\mu_{\mathrm{C}}(P, T) \\
x_{\mathrm{CO}}+x_{\mathrm{CO}_{2}}+x_{\mathrm{C}} & =W_{\mathrm{C}} \\
x_{\mathrm{CO}}+2 x_{\mathrm{CO}_{2}} & =W_{\mathrm{O}}
\end{aligned}
$$

Here $x_{\mathrm{CO}_{2}}$ is the concentration of $\mathrm{CO}_{2} . W_{\mathrm{C}}$ is the concentration of the element $C$ in the system. In words the equations say: balance the chemical potentials while keeping the total moles of each element fixed. CHEETAH is nothing but a complicated chemical potential balancer.

\subsection{FY94 Accomplishments}

A list of accomplishments made in the CHEETAH project during FY94 is given below. A more detailed description of code features is given in the CHEETAH 1.0 User's Manual[1]. CHEETAH 1.0 was released to beta testers in the Office of Munitions Technical Coordinating Group in July 1994. Since then, CHEETAH has been released to more than 30 beta testers at DoD and DOE laboratories, industry, and academia. Work on CHEETAH has fallen into one of three categories: enhanced user convenience, new algorithms, and new capabilities.

\subsubsection{User convenience}

- CHEETAH has been extensively documented. A 140 page user's manual[1] was prepared and professionally published. The manual includes a tutorial to help new users.

- We have started an electronic mail hotline for CHEETAH users. The hotline is an informal discussion group, where users can send questions or suggestions. More than 80 pages of discussions have been logged on the hotline since July.

- CHEETAH has a single command standard run. It is now possible to run CHEETAH by giving only the composition and density.

- The input routines of TIGER have been re-written. Longer, more logical, names can be used. Input files now accept comments.

- CHEETAH has a simple macro facility for frequently used commands.

- CHEETAH supports a startup file that loads initialization commands automatically.

- The output of CHEETAH can be exported to a spreadsheet file. This file is readable by Excel, Quattro, etc.

- CHEETAH supports user-defined and relative units for input.

- CHEETAH produces a single sheet summary of a run. The summary sheet has all the information that most users require.

- CHEETAH has a reactant database containing most frequently used explosives and binders. This saves the user the inconvenience of looking up thermodynamic constants. 
- Product libraries now have titles. This allows the library used to be clearly identified in the output files.

- CHEETAH supports multiple product libraries.

\subsubsection{Algorithmic improvements}

- CHEETAH has a new solver for chemical equilibrium that is more reliable than TIGER's.

- Difficulties with freezing concentrations in TIGER have been fixed.

- CHEETAH has a new Hugoniot solver that is faster and more robust than TIGER's.

- CHEETAH has a new C-J solver that is more reliable than TIGER's.

- CHEETAH has a new solver for solid-liquid phase coexistence.

- CHEETAH can be dynamically resized to run large problems or accomodate small computers.

- A version of CHEETAH for MS-Windows was developed. The program is not limited to $640 \mathrm{~K}$ of memory and can be resized to take advantage of available memory.

\subsubsection{New capabilities}

- CHEETAH has wide variety of thermodynamic point types. Most notably, T-S points are available for freezing along an adiabat.

- CHEETAH has a JWL fitting program built in. There is also a stand-alone program for fitting experimental cylinder test data to a JWL.

- CHEETAH estimates the portion of the detonation energy that is available for doing mechanical work.

- CHEETAH estimates cylinder test wall velocities for comparison with experiment.

\subsubsection{Improved equation of state}

The BKW equation of state has been reparametrized for $\mathrm{CNOH}$ explosives. The reparametrization was done through exhaustive computer search techniques. The new parameters are capable of generating reliable detonation velocities, C-J pressures, adiabat energies, and final energies of detonation.

\section{Code improvements in CHEETAH 1.0}

The following sections describe changes to TIGER made in developing CHEETAH. 


\subsection{New input routines}

TIGER was written before FORTRAN had character string types. Because of this, the authors translated character strings into floating point numbers. This had nightmarish consequences for the readability of the code. For instance, the string 'ALPHA' appears as the number 1526302215.0 in TIGER. It also had subtle consequences for the user. Single-precision versions of TIGER, (like Dan Calef's PC version) were limited to recognizing only the first three characters of a string. This made the creation of constituent libraries very error-prone, since one had to be sure that no two constituents shared the same first three letters.

To solve this problem, a set of modifications were made to the entire code that replaced all the input with a modern token-based input system. While commands may be abbreviated to three letters, further letters are now significant. Spaces in words are now recognized. Although commands can be abbreviated, user-defined names cannot. This is to help reduce errors in library files.

CHEETAH keeps a "dictionary" of strings that it has learned in previous runs and while loading the library, in the file string.in. An ' $\&$ ' is provided as a continuation character for long lines. The ' $\&$ ' must be the last character on the line. Any input line beginning with a "\#" character is a comment. Comments are echoed into the output file but are otherwise ignored.

\subsection{Load file command}

The command

load file, myfile.in

instructs CHEETAH to execute the commands contained in myfile. in. After the commands are executed, it returns to running commands from the original input file. The load file command is particularly useful in conjunction with relative units (see below).

\subsection{Startup file}

Any commands in the file startup. in will be executed before the regular input file. Favorite input units, equation of state set commands, or frequently used reactants are good candidates for inclusion into the startup file.

\subsection{Spreadsheet output}

CHEETAH can create an auxiliary output file consisting of specified thermodynamic variables, in a form readable by most spreadsheets and plotting packages. For example,

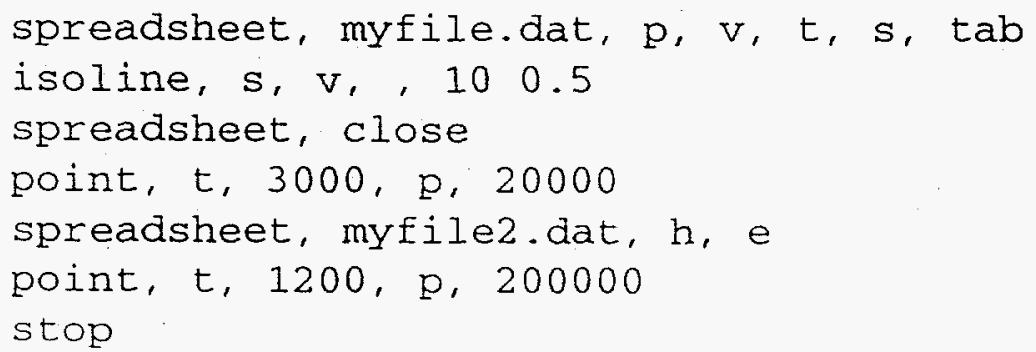


specifies that a spreadsheet file myfile. dat be created, containing values of $p, v, t$, and $s$. The columns in the file are to be separated by tabs. Other separator options are comma and space. By default, columns are separated by commas. The first argument to spreadsheet must be either a filename or close.

The close command is necessary to make sure that unwanted points do not end up in the spreadsheet file. In the above example, the point at $3000 \mathrm{~K}$ and 20000 atm would be put in the spreadsheet file were it not for the close command. Spreadsheet commands can follow one another without an intervening close, in which case the set of variables used in the first spreadsheet command is inherited by the second. Spreadsheet can report the following variables: $\mathrm{p}, \mathrm{t}, \mathrm{v}, \mathrm{h}, \mathrm{s}, \mathrm{e}, \mathrm{v}_{\mathrm{gas}}, \alpha, \beta, C_{v}$, and $\kappa$. In addition, CHEETAH can report the concentrations of selected constituents, by giving the constituent name.

\subsection{Improvements to the freeze command}

The freeze command in TIGER used an algorithm that was susceptible to roundoff errors. The consequence of this to the user was that TIGER would frequently refuse to freeze concentrations, so that freezing had to be done in multiple steps. The freezing algorithm was modified in CHEETAH to make it more reliable.

\subsection{Reformulated concentration solver}

For every calculation type (point, $c-j$, isoline), TIGER solves at least once for the concentrations of the non-frozen constituents at a specified thermodynamic state (e.g. P,T). The original solver would often fail to converge. This occurred for two reasons: the two-step solver used in TIGER could get trapped in local minima that a single-step solver would avoid. Secondly, the Newton solvers in TIGER used a poor backtracking mechanism that often led to poor convergence.

In the two-step solver the concentrations were adjusted at fixed $\eta$ values (the $\eta$ were related to chemical potentials), then the $\eta$ s were adjusted. The problem with the two-step scheme is that if a difficult or unphysical set of $\eta$ 's was chosen, the inner concentration solver would fail.

The $\eta \mathrm{s}$ have now been eliminated as basic variables in CHEETAH. Instead, the log concentrations, temperature, and specific volume are adjusted simultaneously in a single solver. The new solver implements a different set of equations than TIGER. The new thermo solver also incorporates bounds so that no concentration can exceed the maximum allowed by stoichiometry. The new solver is more robust than the TIGER solver, and maintains the exceptional speed of the original TIGER solver.

\subsection{New point types}

CHEETAH allows the user to specify the thermodynamic state ("point") by giving any two functions from the list (p,v,t,e,h,s,a,Hugoniot). See section 2.9 for information on Hugoniot points. Most notably, T-S points can be given. This should be useful for freezing along an isentrope. Other exotica, such as H-S points, are possible. Currently, the new point types require that you first run an ordinary point, such as p-t, p-v, etc. Sometimes the state specified does not exist (e.g., not all $p-v$ states are attainable), in which case CHEETAH will be unable to find a solution. 


\subsection{New isoline types}

Corresponding to the new point types, isoline accepts new variables. Any thermodynamic variable from the list (p,v,t,e,h,s,Hugoniot) can be held constant, while any other variable from the list can be changed. Isentrope integrals are only calculated for p-s or v-s lines, as in TIGER.

\subsection{New Hugoniot solver}

TIGER used a simple iteration scheme to find a point on a shock Hugoniot. The same iteration was used within the C-J solver. Unfortunately, iterative methods don't always converge; the C-J and Hugoniot solvers in TIGER often ran into difficulties. CHEETAH implements a Hugoniot point in a different way than TIGER: rather than solving for a successive number of $(p, v)$ points, it uses the equation

$$
e_{1}-e_{0}=\left(p_{1}+p_{0}\right)\left(v_{0}-v_{1}\right) / 2
$$

as an implicit definition of the thermodynamic state. Thus, saying that we are on the Hugoniot plays the same role in CHEETAH as saying that the pressure is $100 \mathrm{kBar}$.

The reference state $\left(p_{0}, v_{0}, e_{0}\right)$ is specified through the new hug 0 command. It behaves very similarly to the specification of the reference state in TIGER's $c-j$ or hugoniot command.

\subsection{New C-J solver}

CHEETAH has a new C-J solver that should be more robust than TIGER's solver. CHEETAH's solver takes advantage of the hugoniot point type. A series of $p$, hugoniot calculations are performed until a pressure is found that satisfies the equation

$$
v=v_{0}\left[\left(1-p_{0} / p\right) / \kappa+1\right]^{-1}
$$

is satisfied. Here, $v$ is the volume at the specified pressure on the Hugoniot line, and $\kappa$ is the adiabatic exponent. The detonation pressure is assumed to be between the constant volume explosion point and $500 \mathrm{kBar}$. Using these points as brackets, it finds the root of Eq. (2) with Brent's method.

The $c-j$ command also has a new syntax. The reference point on the Hugoniot is specified with the hug 0 command described in Sec. 2.9. The $c-j$ command doesn't take any arguments. For example,

$$
\underset{c-j}{\operatorname{hug} 0, p, 1, \text { rho, } 1.890}
$$

\subsection{User-defined units}

Units to use when entering pressures or volumes can be specified with the commands

$$
\begin{array}{ll}
\text { units, } & p, 100 \\
\text { units, } & v, 2 \\
\text { units, } & p,
\end{array}
$$


The first command sets the input unit of pressure to be $100 \mathrm{Atm}$. The second command sets the input unit of relative volume to be $2 \mathrm{cc} / \mathrm{gm}$. The third command sets the unit of pressure to be the current pressure. This is particularly useful when specifying pressures or volumes relative to the last thermodynamic state in the calculation. The units in the output file are unaffected by the units command.

Units can be set for $p, v, \rho, E$ and $T$. If $E$ units are specified, all quantities with the dimensions of energy $(E, H, G)$ are input with the new $E$ units. The unit of $S$ is the unit of $E$ divided by the unit of $T$.

There are two special ways to set the unit of volume. The command units, sv indicates that the standard volume is the new input unit of volume. The standard volume is defined as:

$$
V_{s}=\sum_{i} x_{i} V_{i}
$$

where $x_{i}$ is the mole fraction of reactant $i$ and $V_{i}$ is the molar volume of the reactant in its standard state. The molar volume in the standard state is usually the same as the inverse of the TMD divided by the molecular weight, so $V_{s}$ can generally be regarded as the TMD of the reactant mixture.

The second way to define the unit of volume is through the command units, $v 0$. In this case the unit of volume is set to be the shock Hugoniot reference volume $v_{0}$. This volume should have been previously set through the hug 0 command or the standard run command. The v0 command gives volumes in reference to the initial explosive volume; therefore it is of particular use in specifying expansion volumes.

\subsection{Summary sheet output}

A file summarizing the run can be generated by using the command:

summary, myfile.sum

where myfile. sum is the name of the summary file. The file summary.out is created as the default summary file if the summary command is not given. The summary file contains the initial composition, C-J parameters, and the volumes and energies of any subsequent S-V points. The $\mathrm{S}-\mathrm{V}$ points correspond to cylinder test results. Subsequent summary commands close the previous summary file. The summary command with a blank argument closes the current summary file.

\subsection{JWL.Fitting}

CHEETAH will automatically determine a JWL fit to the C-J point and the adiabat. The appropriate command is $j w l f i t$. This command should be given after finding the C-J point and at least 3 points along the adiabat. This should be followed by the det energy command, which finds the detonation energy. More than 3 points along the adiabat can also be used, in which case the JWL fitter will find the best possible fit to the points. jwlfit takes a single argument, which is the number of cylinder runs to use in the fit. At least three runs must be used. By default, all the previous cylinder runs are used. 


\subsection{Det energy command}

This command is used to find the detonation energy. The energy of detonation is broken out into a mechanical and a thermal part.

\subsection{Standard run command}

The standard CHEETAH run performed at LLNL can be executed with the standard run command. The syntax is:

standard run, rho, 1.82

where 1.82 is the density of the formulated explosive. Without the rho argument, standard run assumes that the density is equal to TMD. The command first finds the C-J point, assuming a reference pressure of $1 \mathrm{~atm}$ for the Hugoniot. It then proceeds along the adiabat, calculating points at relative volumes equal to $2.2,4.1,6.5,10.0$, and 20.0 . The composition is frozen at $1800 \mathrm{~K}$ along the adiabat. The energy of detonation is then found and finally, the adiabat points are fit to a JWL equation of state.

The standard run can be customized by modifying the file config/standard.in. The first line of the standard. in file is the number of points to calculate along the adiabat. Next follows the relative volumes at each of the points. Next the freezing temperature is given; enter 0.0 if freezing is not desired. The last line of the standard. in file controls whether JWL fitting is done or not. A 1 indicates that the adiabat is to be fit to a JWL equation of state, while a 0 indicates that fitting is not to be done.

\subsection{Formula library}

The old for, ... command to specify the reactant formulas is no longer necessary. Instead, these commands have been put into a database file config/formula.in. When the components command is given, the database is searched for the appropriate formula. The first line of the database gives a title that will be echoed to the main output and summary files. Formulas in the database must begin with the command for, starting with the first character in the line. Longer versions of the command (e.g. formula, . ) cannot be used in the database file. The formula command can be used in the input file to override database values. The use of a formula command in the input file discontinues searching of the database for all formulas.

\subsection{Configuration directory}

All startup and configuration files are now put in a configuration directory. By default, the directory is config.

\subsection{New mixture solver}

The mixture solving and detection routines from TIGER have been replaced with new routines in CHEETAH. The old subroutine CHKPHA has been replaced by the $\mathrm{C}$ subroutine checkphase. The following algorithm is used to check the condensed phase assumptions: if condensed phase 
assumptions are violated, new assumptions are generated which are correct for the current $(T, P)$ values. The thermodynamic state (and hence $(T, P)$ ) are recalculated using these assumptions. If the assumptions are again incorrect, a unique set of new assumptions are generated, until all possible assumptions are exhausted. At this point, checkphase() concludes that the state of the system must be a solid-liquid mixture. checkphase() then looks through all previously tried guesses to find a candidate for the melting compound.

The melting compound is identified when the following conditions are met: there exists two guesses identical in all assumptions except the ith. For these two guesses the chemical potential conditions are satisfied for all but the ith condition, which is violated in both of the guesses. It is then concluded that the ith condensed constituent should be in a mixture state.

The liquid fraction $f_{l}$ is then determined in a new subroutine, called mixmaster. This subroutine uses Brent's method to find the value of $f_{l}$ such that the chemical potential of the solid is equal to that of the liquid for condensed constituent $i$. In general, the mixmaster() routine is somewhat slower than the iteration method used in TIGER, but it should be more reliable.

\subsection{Dynamic memory allocation}

The size of internal arrays in CHEETAH can be easily changed by the user. Large numbers of products or reactants can be run on computers with sufficient memory (RAM), while CHEETAH can be kept small for PC's or other systems with little memory. CHEETAH's array sizes are controlled by the file config/a110c. in. The first number is the maximum number of gaseous products (called constituents in the language of TIGER) The second is the maximum number of condensed products. The third number is the maximum number of elements (called components in TIGER). Finally the fourth number is the maximum number of reactants. The al1oc. in file can be edited to change the default array sizes, and the changes then take effect upon running CHEETAH.

\subsection{Library file command}

CHEETAH can keep track of more than one pre-compiled library file at a time through the use of the library file command. The command library file, mylib. chl should be placed in the library prior to running it with CHEETAH. You then run CHEETAH on a library file:

$$
\text { cheetah library. in library.out }
$$

The library fil e command specifies that the compiled library will be stored in mylib. chl, rather than the default cheetah. chl.

Then, library files may be selected with the command library file, mylib.chl in the input file or config/startup. in. If the library file command is not used, CHEETAH will store and read the library in the file cheetah. chl.

\subsection{Library titles}

Product libraries can now be titled. CHEETAH prints the title of the current library in the output and summary files. Naming libraries is highly recommended as a mechanism for documenting 
the library associated with a particular output file, especially when using the library file command above to switch between libraries.

To give a library a title, enter the command

title, an example library

immediately after the start of library command in the library input file. This title is recorded in the binary library file and is reported for each subsequent CHEETAH run using that library.

\subsection{Details command}

By default, CHEETAH shows fewer thermodynamic functions than TIGER did. By using the details command, the reporting of these functions is restored. After the details command, the following information is shown: the constant volume heat capacity, the hydrodynamic constants $\alpha, \beta$, and $\kappa . \kappa$ was called ADEXP in TIGER.

The definitions are as follows:

$$
\begin{aligned}
C_{V} & \equiv\left(\frac{\partial e}{\partial T}\right)_{V} \\
\alpha & \equiv \frac{1}{p}\left(\frac{\partial e}{\partial v}\right)_{p} \\
\beta & \equiv \frac{1}{v}\left(\frac{\partial e}{\partial p}\right)_{v} \\
\kappa & \equiv-\left(\frac{\partial \ln p}{\partial \ln v}\right)_{s}=\frac{\alpha+1}{\beta}
\end{aligned}
$$

\section{BKWC: A new parametrization of the BKW equation of state}

A database containing mostly cylinder test data for 42 explosives composed of $C, N, O, H, F$, and $\mathrm{Cl}$ was collected. The resulting detonation velocities, $\mathrm{C}-\mathrm{J}$ pressures, adiabat energies, and total energies of detonation were used to reparametrize the BKW equation of state for the CHEETAH thermochemical code. The resulting parameter set, referred to as BKWC, reproduces detonation velocities and adiabat energies better than previous BKW parametrizations. The BKWR equation of state is found to be slightly better in yielding C-J pressures.

\subsection{Introduction}

C-J detonation theory [4] implies that the performance of an explosive is determined by thermodynamic states-the C-J state and the C-J adiabat. Thermochemical codes use thermodynamics to calculate these states. and hence obtain a prediction of explosive performance. The allowed thermodynamic states behind a shock are intersections of the Rayleigh line (expressing conservation of mass and momentum), and the shock Hugoniot (expressing conservation of energy). C- $J$ theory states that a stable detonation occurs when the Rayleigh line is tangent to the shock Hugoniot.

This point of tangency can be found, given that the equation of state $P=P(V, T)$ of the products is known. The chemical composition of the products changes with the thermodynamic state, so 
thermochemical codes must simultaneously solve for state variables and chemical concentrations. This problem is relatively straightforward, given that the equation of state of the gaseous and solid products is known.

One of the most difficult parts of this problem is accurately describing the equation of state of the gases. The Becker-Kistiakowski-Wilson (BKW) $[5,6]$ gaseous equation of state (EOS) has a long and venerable history in the explosives field. The BKW EOS has the following form:

$$
\begin{aligned}
\frac{p v}{n R T} & =1+x \exp (\beta x) \\
x & =k / v(T+\theta)^{\alpha} \\
k & =\kappa \sum_{i=1}^{n} n_{i} k_{i}
\end{aligned}
$$

Here, $p$ is the pressure, $v$ is the molar gas volume, and $R$ is the gas constant. $\dot{\alpha}, \beta$, $\kappa$, and $\theta$ are adjustable equation of state parameters. $n_{i}$ is the mole fraction of species $i$ and the $k_{i}$ are covolumes.

Despite its simplicity and lack of rigorous derivation, the BKW EOS is used in many practical applications in the explosives field. There have been a number of different parameter sets proposed for the BKW EOS. Mader[6] used two BKW parameter sets for high oxygen and high carbon explosives. Finger et. al.[7] proposed the BKWR (for renormalized) parameters, while Baer and Hobbs[8] proposed the BKWS parameter set. The work of Baer and Hobbs was critical in reviving interest in the BKW equation of state and extending its use to a wide range of inorganic compounds. Kury and Souers[9] have critically reviewed these equations of state by comparing their predictions to a database of cylinder tests performed at Lawrence Livermore National Laboratory (LLNL). They concluded that BKWR gave the best predictions for detonation velocities and CJ pressures, but failed to reproduce the shape of the adiabat without the use of empirical scaling factors. BKWS gave better results for the adiabat, but suffered from poorer prediction of detonation velocites.

We have undertaken a project to update and extend the TIGER[2] thermochemical code at LLNL. The new code, named CHEETAH[1], currently implements the BKW and JCZ3[10] equations of state. More sophisticated equations of state will likely be added in the future. As a point of departure, however, we decided to explore the limits of accuracy possible within the BKW framework. To that end we have employed a modern stochastic optimization algorithm to find the global parameters and covolumes that best match available experimental data. We refer to the new parameters as BKWC (for CHEETAH). The performance of the BKWC equation of state will serve as a practical test of the utility of other equations of state that we will pursue in the future.

Caution must be employed when comparing predicted $\mathrm{C}-\mathrm{J}$ properties from an equilibrium code and experimental data. According to Zeldovich-Von Neumann-Doering (ZND) theory [4], the C-J state is attained behind a planar detonation wave traveling through an infinite medium after an infinite period of time. Of course, real experiments are always carried out on finite systems. Finite system size reduces the effective time available for equilibration; the developing C-J state is disrupted by rarefaction waves. Thus the C-J state is never exactly attained in any real experiment. Notionally we may say that the C-J state is replaced by a frozen "sonic point" $[11,12]$ where the condition $D=U_{p}+c$ is achieved, but chemical equilibrium is not completely achieved. Even the concept of a sonic point, however, is simplified. Since geometric (e.g. sample shape and confinement) effects determine the behavior of an explosive, its properties cannot be described by a single thermodynamic state, but rather by a spatial distribution of states.

We hope to address some of the above issues in the near future. For the present time, however, we will cease to distinguish between the sonic point and the C-J state. This is consistent with our 
desire to make a predictive model that can be directly compared to experimental data.

\subsection{Method}

We have collected a database of explosive performance properties, mostly consisting of cylinder tests performed at LLNL[13, 14, 15]. Table 1 gives the composition, heat of formation, and density of the compounds in the database. A wide range of stoichiometries was chosen. Most explosives of wide practical utility are included in the database. Carbon rich (TATB, TNT) as well as high oxyygen (TNM) explosives were included. The database contains compounds lacking hydrogen (BTF,TNM) and carbon (RX-23-AA). There are several non-ideal explosives, most notably ANFO. Although ANFO has notoriously strong size dependences, the data reported for this explosive is based on a large scale underground explosion of more than a kiloton[16]; we expect the ANFO values to be fully size converged.

We started with EKWS as an initial parameter set, since it gives good results without scaling factors. The BKWS library, however, contains a large number of product species. For instance, there are 61 product species containing $\mathrm{C}, \mathrm{N}, \mathrm{O}$, and $\mathrm{H}$. While it is always safer to do calculations with a large number of product species than it is to have too few, in practice the use of so many products slows calculations dramatically. Since we envisioned the BKWC library as an everyday tool for explosive formulators, it was necessary to determine the minimal number of products needed to obtain good results for "usual" explosives.

To this end, we ran calculations on all the CNOH compounds in the data base. We used BKWS values for $\alpha, \beta, \kappa$, and $\theta$ given in Table 2. In each calculation the C-J state was found, then points along the explosive adiabat at 2.2, 4.1, and 6.5 relative volumes were found. A further expansion was then done along the adiabat to 1 ATM or $298 \mathrm{~K}$, whichever condition came first. We define this point to be the end of the adiabat. The reason for the $298 \mathrm{~K}$ criterion is that $\mathrm{BKW}$ equations of state occasionally predict very cold (100K or below) product gases after expansion. The heat capacity fits used in CHEETAH break down in this regime. After the end of the adiabat was found, we performed a final calculation at $298 \mathrm{~K}$ and 1 ATM.

For each $\mathrm{CNOH}$ product in the Sandia library, we found the maximum concentration that the product obtained across all the explosives and all thermodynamic states. The resulting maximum concentrations are reported in Table 3. As might be expected, $\mathrm{CO}_{2}, \mathrm{H}_{2} \mathrm{O}$, and $\mathrm{NH}_{3}$ are among the most common species. Radicals never achieved significant concentrations, with the exception of $\mathrm{CH}_{3}$. We kept all species attaining a maximum concentration of $0.05 \mathrm{~mol} / \mathrm{kg}$ or greater in the BKWC library. This yields 17 product species, a number modestly greater than the 12 species used in BKWR, but much less than the 61 species of BKWS. A similar procedure was followed to determine the necessary $\mathrm{F}$ and $\mathrm{Cl}$ products.

We repeated the runs with the abbreviated BKWS library. By comparing the results of the abbreviated BKWS with the full BKWS, we found the fractional root mean square (RMS) error in the pressure, volume, temperature, and energy across all the thermodynamic states in the calculation. The RMS pressure error was $0.05 \%$, the temperature error was $0.07 \%$, the volume error was $0.03 \%$, and the energy error was $0.11 \%$. These values are much less than typical disagreements between thermochemical code results and experiment; the abbreviated library does not cause significant errors.

Having determined a minimal acceptable set of product species, we now discuss the adjustment of parameters to match the experimental database. Calculations of the C-J state and the adiabat 


\begin{tabular}{|c|c|c|c|c|c|c|c|c|}
\hline Name & C & $\mathrm{H}$ & $N$ & $\mathrm{O}$ & $\mathrm{F}$ & $\mathrm{Cl}$ & $\rho(\mathrm{g} / \mathrm{cc})$ & $H_{f}(\mathrm{kcal} / \mathrm{mol})$ \\
\hline $\mathrm{BTF}$ & 6 & & 6 & 6 & & & 1.85 & 144.5 \\
\hline $\mathrm{HMX}$ & 4 & 8 & 8 & 8 & & & 1.89 & 17.9 \\
\hline $\mathrm{HMX} 2$ & 4 & 8 & 8 & 8 & & & 1.18 & 17.9. \\
\hline HNB & 6 & & 6 & 12 & & & 1.97 & 15.7 \\
\hline LX14 & 15.7 & 30.1 & 26.7 & 27.5 & & & 1.83 & 4.4 \\
\hline PETN & 5 & 8 & 4 & 12 & & & 1.76 & -128.7 \\
\hline PETN2 & 5 & 8 & 4 & 12 & & & 1.50 & -128.7 \\
\hline PETN3 & 5 & 8 & 4 & 12 & & & 1.26 & -128.7 \\
\hline TNM & 1 & & 4 & 8 & & & 1.65 & 118.8 \\
\hline HNS & 14 & 6 & 6 & 12 & & & 1.66 & 18.7 \\
\hline HNS2 & 14 & 6 & 6 & 12 & & & 1.40 & 18.7 \\
\hline HNS3 & 14 & 6 & 6 & 12 & & & 1.20 & 18.7 \\
\hline HNS4 & 14 & 6 & 6 & 12 & & & 1.00 & 18.7 \\
\hline NM & 1 & 3 & . 1 & 2 & & & 1.13 & -27.0 \\
\hline RX-23-AA & & 51.8 & 29.1 & 19.1 & & & 1.42 & -27.7 \\
\hline TATB & 6 & 6 & 6 & 6 & & & 1.83 & -36.9 \\
\hline TNT & 7 & 5 & 3 & 6 & & & 1.63 & -17.8 \\
\hline $\mathrm{RX}-26-\mathrm{AF}$ & 20.4 & 28.4 & 25.9 & 25.3 & & & 1.84 & -20.6 \\
\hline ANFO & 47.1 & 19.7 & 29.6 & 3.6 & & & 0.80 & -86.4 \\
\hline QM100R & 7.3 & 44.8 & 18.9 & 29.0 & & & 1.51 & -64.8 \\
\hline DP12 & 20.0 & 40.0 & 13.3 & & 26.7 & & 1.26 & -48.0 \\
\hline AFX902 & 10.0 & 35.9 & 35.0 & 17.5 & 1.7 & & 1.74 & -30.9 \\
\hline FEFO & 5 & 6 & 4 & 10 & 2 & & 1.61 & -177.5 \\
\hline FMl & 20.0 & 30.2 & 13.3 & 33.2 & 3.2 & & 1.51 & -159.7 \\
\hline $\mathrm{PF}$ & 6 & 2 & 3 & 6 & 1 & & 1.83 & -62.0 \\
\hline $\mathrm{RX}-36-\mathrm{AH}$ & 22.3 & 17.7 & 29.0 & 29.0 & 2.1 & & 1.83 & 51.6 \\
\hline$R X-41-A B$ & 15.9 & 20.2 & 28.6 & 33.3 & 2.1 & & 1.86 & -30.1 \\
\hline LX17 & 25.4 & 24.2 & 23.8 & 23.8 & 2.2 & 0.6 & 1.91 & -62.9 \\
\hline $\mathrm{RX}-27-\mathrm{AD}$ & 37.3 & 12.3 & 23.7 & 23.7 & 2.5 & 0.7 & 1.64 & 61.8 \\
\hline RX-45-AA & 17.2 & 24.5 & 40.3 & 16.1 & 1.5 & 0.4 & 1.75 & 4.8 \\
\hline RX-47-AA & 27.6 & 17.7 & 25.9 & 25.9 & 2.4 & 0.6 & 1.82 & -8.0 \\
\hline $\mathrm{RX}-48-\mathrm{AA}$ & 30.2 & 14.6 & 23.6 & 28.4 & 2.5 & 0.7 & 1.85 & 8.5 \\
\hline
\end{tabular}

Table 1: Composition, density, and heat of formation of compounds in the performance database.

For mixtures, the composition is given as mole fractions.

$\begin{array}{lllll}\text { EOS } & \alpha & \beta & \kappa\left(\mathrm{cc} K^{\alpha}\right) & \theta(\mathrm{K}) \\ \text { BKWR } & 0.5 & 0.176 & 11.80 & 1850 \\ \text { BKWS } & 0.5 & 0.298 & 10.50 & 6620 \\ \text { BKWC } & 0.5 & 0.403 & 10.86 & 5441\end{array}$

Table 2: BKW parameters used in the calculations 


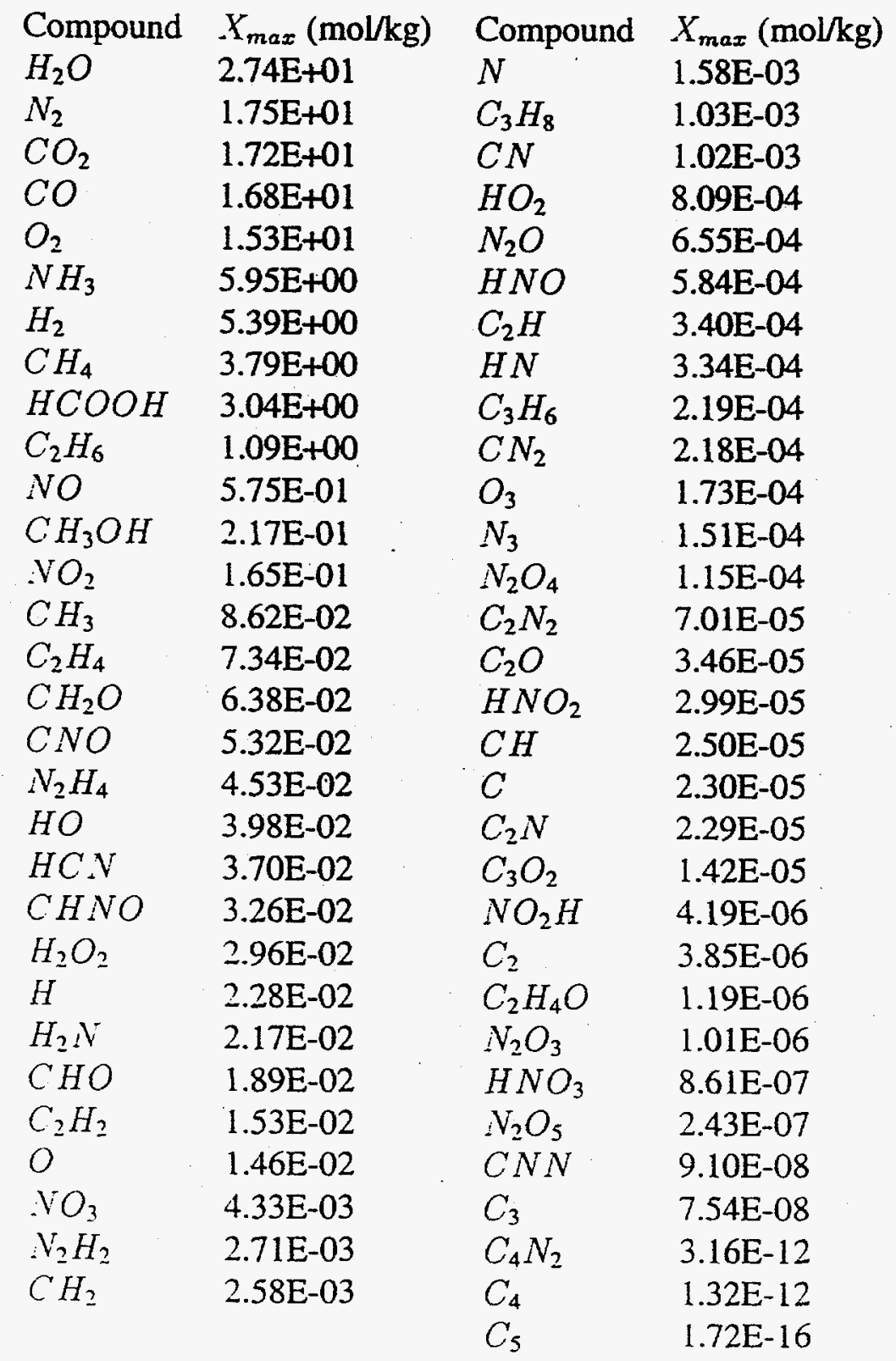

Table 3: Maximum concentrations $\left(X_{m a x}\right.$ ) found with BKWS when performing C-J and adiabat calculations on all compounds in the performance database. 
were performed as described above, with the exception that concentrations were frozen at a temperature $T_{f}$. The presence of substantial concentrations of $\mathrm{CO}$ in detontion products, as found by Ornellas[17], indicates that thermodynamic equilibrium is not fully achieved through a typical adiabatic expansion. It has been standard practice at LLNL to freeze concentrations along the adiabat at $1800 \mathrm{~K}$. In the present treatment, we take the freezing temperature to be an adjustable parameter.

The performance data we used is given in Table 4. Most of the data is collected from cylinder tests, with the exception of ANFO and QM100R. We adjusted BKW parameters and covolumes to find the "best fit" to the experimental data. Standard pressure reference data (heat of formation, entropy of formation, and heat capacity as a function of temperature) were not adjusted. BKWC uses the same standard pressure data as BKWS; which in turn was based on JANAF table data[18].

Some thought must be put into the definition of the objective function (i.e. the error to be minimized) in order to obtain the parameter set that best describes physical reality. The difficulty in matching to experimental data is that different quantities (e.g. detonation velocity, C-J pressure) can have disparate error bars. If the difference between experiment and theory was minimized without regard to the underlying error bars, those quantities with largest error bars would dominate the fitting process. In effect we would be trying to reproduce experimental errors. The problem is compounded by the fact that experimental error bars are often difficult to find. The relevant error is not how well a quantity in a particular finite sized experiment was measured (e.g. the detonation velocity in a 2 in. cylinder), but rather how well the finite size measurement approximates an infinite system. After consulting previous work, we arrived at the following rough error estimates.

Detonation velocities are determined from pin rings attached to the cylinders. For 25 and $50 \mathrm{~mm}$ cylinders of $300 \mathrm{~mm}$ length, the pin rings are set at 90 and $290 \mathrm{~mm}$. The measured velocity is thus across a distance of $200 \mathrm{~mm}$, so that the accuracy is much better than $1 \%$. The difficulty arises in relating cylinder velocities to infinite diameter theoretical predictions. TATB, for example, has a strong detonation velocity size dependence. The difference between $D$ at $50 \mathrm{~mm}$ diameter and $100 \mathrm{~mm}$ diameter is $0.6 \%$ [19]. Since our data is at $50 \mathrm{~mm}$, we took $1 \%$ as an error estimate.

For the C-J pressure, we estimated an error of $10 \%$. This is based on a comparison of C-J pressure measurements for PETN using various experimental techniques[12]. 5\% errors were estimated for adiabat energies. This was based largely on the use of streak camera data, which typically has velocities of about $2 \pm 0.1 \mathrm{~mm} / \mu \mathrm{s}$. This error can be reduced to perhaps $\pm 1 \%$ with the use of Fabry-Perot interferometry.

Total energies of detonation, as determined by bomb calorimetry[17], have a variance between runs of 0.5 to $1 \%$. The error in the adiabat endpoint, however, is probably higher. Some explosives (e.g. TNT) show variable results according to confinement. Thus an overall error of $3 \%$ was used. Some energies of detonation were determined through fitting the cylinder data to a JWL equation of state. Comparison with Ornellas' [17] data yielded an error estimate of 5\%.

Our approach was to optimize the EOS to give results that are within experimental error bars. To this effect, we defined the discrepancy between a theoretical and a measured quantity to be:

$$
\begin{array}{ll}
d=0 & |(T-E) / E|<F \\
d=|(T-E) / E|-F & |(T-E) / E| \geq F
\end{array}
$$

Here $T$ is the theoretical value, $E$ is the experimental value, $F$ is the estimated fractional experimental error bar, and $d$ is the discrepancy. The discrepancy is defined to be zero if we are inside 


\begin{tabular}{|c|c|c|c|c|c|c|c|c|c|}
\hline Compound & I & $\mathrm{D}(m m / \mu s)$ & $P_{\mathrm{CJ}}(\mathrm{GPa})$ & $E_{\text {cal }}$ & $E_{\text {JWL }}$ & $E(2.2)$ & $E(4.1)$ & $E(6.5)$ & Ref. \\
\hline BTF & 1 & 8.49 & 34.0 & 11.2 & 11.3 & -6.72 & -8.19 & -9.09 & $\mathrm{C}$ \\
\hline $\mathrm{HMX1}$ & 1 & 9.10 & 40.5 & 11.0 & 11.0 & -7.10 & -8.81 & -9.57 & $\mathrm{C}$ \\
\hline $\mathrm{HMX2}$ & 1 & 6.674 & 15.5 & 6.2 & 6.4 & -3.59 & -4.59 & -5.17 & $\mathrm{C}$ \\
\hline HNB & 1 & 9.335 & 43.0 & 13.7 & 13.2 & -7.54 & -9.40 & -10.31 & $\mathrm{C}$ \\
\hline HNSI & 1 & 7.030 & 21.5 & 7.5 & 7.5 & -3.93 & -4.93 & -5.54 & $\mathrm{C}$ \\
\hline HNS2 & 1 & 6.340 & 16.0 & & 5.8 & -3.05 & -3.88 & -4.33 & $\mathrm{C}$ \\
\hline HNS3 & 1 & 5.740 & 11.5 & & 4.6 & -2.40 & -3.06 & -3.54 & C \\
\hline HNS4 & 1 & 5.100 & 7.3 & 3.7 & 3.6 & -1.74 & -2.31 & -2.60 & $C$ \\
\hline NM & 1 & 6.280 & 12.0 & 5.2 & 4.95 & -2.85 & -3.70 & -4.08 & C \\
\hline PETN1 & 1 & 8.274 & 31.0 & 10.3 & & -6.51 & -7.87 & -8.55 & $\mathrm{C}, \mathrm{J}, \mathrm{S}$ \\
\hline PETN2 & 1 & 7.480 & 24.0 & 8.9 & 8.5 & -4.89 & -6.18 & -6.82 & $\mathrm{C}$ \\
\hline PETN3 & 1 & 6.590 & 16.0 & & 7.2 & -3.59 & -4.65 & -5.20 & C \\
\hline RXA23A & 1 & 8.640 & 25.8 & 6.5 & 6.5 & -4.54 & -5.77 & -6.23 & $\mathrm{C}$ \\
\hline ТATB & 2 & 7.580 & 26.0 & 7.3 & & -4.41 & -5.24 & -5.70 & $\mathrm{C}$ \\
\hline TNM & 1 & 6.449 & 15.5 & & 3.6 & -2.61 & -3.19 & -3.45 & $\mathrm{C}$ \\
\hline TNT & 2 & 7.070 & 20.5 & 7.1 & 7.1 & -3.82 & -4.88 & -5.36 & $\mathrm{C}$ \\
\hline LX14 & 1 & 8.800 & 37.0 & & 10.8 & -6.74 & -8.19 & -8.65 & $\mathrm{C}, \mathrm{J}$ \\
\hline $\mathrm{RX} 26 \mathrm{AF}$ & 1 & 8.240 & 34.0 & & 9.2 & -5.63 & -6.66 & -7.09 & $\mathrm{C}, \mathrm{J}, \mathrm{S}$ \\
\hline ANFO & 3 & 4.7 & & & & & & & $\mathrm{~N}$ \\
\hline QM100R & 3 & 7.7 & & & & & & & $\mathbf{N}$ \\
\hline DP12 & 1 & 5.97 & 12.5 & 7.2 & 6.9 & -3.08 & -4.08 & -4.63 & C \\
\hline AFX902 & 1 & 8.344 & 29.0 & & 7.0 & -4.39 & -5.10 & -5.42 & $\mathrm{C}$ \\
\hline FEFO & 1 & 7.451 & 24.5 & 8.3 & 8.2 & -4.68 & -5.91 & -6.57 & C \\
\hline FMI & 1 & 6.569 & 19.0 & & 7.1 & -4.13 & -5.14 & -5.71 & $\mathrm{C}$ \\
\hline PF & 1 & 7.290 & 27.0 & & 7.5 & -5.07 & -6.19 & -6.78 & $\mathrm{C}$ \\
\hline RX36AH & 1 & 8.511 & 33.5 & & 12.0 & -6.45 & -7.83 & -8.60 & $\mathrm{C}$ \\
\hline $\mathrm{RX} 41 \mathrm{AB}$ & 1 & 8.814 & 35.0 & & 10.0 & -6.58 & -7.78 & -8.19 & $\mathrm{C}$ \\
\hline LX 17 & 2 & 7.630 & 26.0 & & 7.0 & -4.53 & -5.32 & -5.69 & C, R \\
\hline RX27AD & 1 & 6.928 & 20.0 & & 7.4 & -3.67 & -4.64 & -5.12 & C \\
\hline RX45AA & 1 & 7.710 & 25.0 & & 7.0 & -4.04 & -4.77 & -5.18 & $\mathrm{C}$ \\
\hline RX47AA & 1 & 7.656 & 26.0 & & 9.3 & -4.72 & -5.87 & -6.35 & $\mathrm{C}$ \\
\hline RX48AA & 1 & 7.760 & 26.3 & & 8.5 & -4.97 & -6.15 & -6.72 & $\mathrm{C}$ \\
\hline
\end{tabular}

Table 4: The LLNL performance database. I is the explosive ideality. 1 indicates an ideal explosive, 2 indicates partially ideal, 3 indicates a strongly non-ideal explosive. $E_{c a l}$ is the total energy of detonation measured by bomb calorimetry. $E_{J W L}$ is the total energy of detonation determined by fitting the adiabat to a JWL equation of state. $\mathrm{E}(x)$ is the adiabat energy at a relative volume of $x$. All energies are reported in $\mathrm{kJ} / \mathrm{cc}$. 
experimental errors. The total error function is the weighted root mean squared discrepancy:

$$
\epsilon=\sqrt{\frac{1}{N} \sum_{i} w_{i}^{2} d_{i}^{2}}
$$

Here, $w_{i}$ is a weight given to each explosive property. We chose weights of $50 \%$ for the detonation velocity, $25 \%$ for the C-J pressure, and $25 \%$ for the adiabat.

Having defined the error function $\epsilon$ that is to be minimized, we next discuss the method used to minimize the error. The parameters involved are the usual BKW parameters: $\alpha, \beta, \kappa, \theta$, the freezing temperature $T_{f}$, and the covolumes $k_{i}$. In addition to the gaseous parameters, the carbon equation of state was adjusted. The motivation for this is that other workers have found that empirical "nonequilibrium" carbon models give better results for detonation properties than rigorous equilibrium carbon models $[3,20,21]$. By "non-equilibrium", we mean that model parameters have been adjusted to empirically take into account the difference between the carbon material formed under detonation and the properties of equilibrium carbon. For instance, the heat of formation of diamond was increased by van Thiel and Ree[22] to empirically account for surface effects in carbon clusters. We have used an extremely simple carbon model. Only a single phase (graphite) is present, the equation of state is:

$$
V=V_{0}+a T+b P
$$

All three parameters $\left(V_{0}, a, b\right)$ were adjusted.

Given a set of parameters, the error function is evaluated by running CHEETAH on all of the problems in the database. We would like to find the parameter set that yields the global minimum error. Most multidimensional minimization algorithms head directly to the nearest local minimum. Stochastic minimizers are better at finding global minima, but require many more function evaluations. We have used the stochastic "record to record travel" algorithm[23]. This algorithm is similar to other stochastic minimizers, such as simulated annealing[24], but it does not require the determination of an annealing schedule. The algorithm makes random steps in each parameter, then accepts the step if it leads to an error that is within a given tolerance of the best run so far. We started with the BKWS equation of state, with the reduced set of species described above. The stochastic minimizer was run about 2000 cycles, which corresponds to 60,000 separate CHEETAH runs.

\subsection{Results}

The optimized parameter set is given in Tables 2 and 5. The freeze temperature was raised from $1800 \mathrm{~K}$ to $2100 \mathrm{~K}$. Freezing is a rough way of incorporating kinetics into a thermochemical code. Intuitively, we can say that reactions where $k_{B} T<E_{a}$ should be frozen, where $E_{a}$ is the activation energy for the reaction. Of course, a host of reactions occur in detonation, each with its own activation energy. Setting the freezing temperature is equivalent to stating an "average" activation energy.

Some care is appropriate when considering the covolumes. The term "covolume" is somewhat of a misnomer. In the high density limit, the covolume acts as an effective molecular volume multiplied by a stiffness factor. In the low density limit, however, covolumes can be related to virial coefficients. There is no general relation, however, between molecular volume and the virial coefficient. Therefore covolumes cannot be uniquely identified with a physical molecular property. 
This is a consequence of the empirical and highly simplified nature of the BKW EOS. It is best to think of covolumes as effective interaction parameters that lie somewhere between the molecular volume and virial limits.

In general the covolumes correlate roughly with molecular volume. $\mathrm{CO}_{2}$; for instance, ?as a large covolume than $\mathrm{CO}$. There are exceptions to this rule, however. For instance $\mathrm{NH}_{3}$ has a i:irger covolume than $\mathrm{CH}_{4}$. The effective carbon model has a $7 \%$ lower specific volume than the BKWS carbon model. The coefficient of thermal expansion and the compressibility have been decreased as well.

\begin{tabular}{|c|c|c|c|}
\hline Parameter & BKWC value & BKWS value & BKWR value \\
\hline$T_{f}(\mathrm{~K})$ & 2145 & 1800 & \\
\hline$V_{0}(\mathrm{cc} / \mathrm{mole})$ & 4.637 & 4.993 & 4.993 \\
\hline$a(\mathrm{cc} / \mathrm{mole}-\mathrm{K})$ & $3.584 \times 10^{-5}$ & $3.963 \times 10^{-5}$ & $3.963 \times 10^{-5}$ \\
\hline$b$ (cc/mole-atm) & $-6.068 \times 10^{-6}$ & $-6.378 \times 10^{-6}$ & $-6.378 \times 10^{-6}$ \\
\hline \multicolumn{4}{|l|}{ Covolumes: } \\
\hline $\mathrm{H}_{2} \mathrm{O}$ & 188 & 376 & 270 \\
\hline$N_{2}$ & 374 & 376 & 404 \\
\hline $\mathrm{CO}_{2}$ & 511 & 663 & 610 \\
\hline CO & 372 & 614 & 440 \\
\hline $\mathrm{O}_{2}$ & 306 & 316 & 325 \\
\hline $\mathrm{NH}_{3}$ & 550 & 418 & 384 \\
\hline $\mathrm{H}_{2}$ & 270 & 153 & 98 \\
\hline $\mathrm{CH}_{4}$ & 420 & 493 & 550 \\
\hline $\mathrm{CH}_{2} \mathrm{O}_{2}$ & 737 & 865 & 722 \\
\hline $\mathrm{C}_{2} \mathrm{H}_{6}$ & 1095 & 832 & NA \\
\hline NO & 394 & 394 & 386 \\
\hline $\mathrm{CH}_{3} \mathrm{OH}$ & 649 & 800 & 800 \\
\hline $\mathrm{NO}_{2}$ & 1159 & 626 & $\mathrm{NA}$ \\
\hline $\mathrm{CH}_{3}$ & 732 & 501 & NA \\
\hline $\mathrm{C}_{2} \mathrm{H}_{4}$ & 533 & 834 & NA \\
\hline $\mathrm{CH}_{2} \mathrm{O}$ & 727 & 795 & NA \\
\hline$H F$ & 684 & 654 & 389 \\
\hline CHFO & 810 & 770 & NA \\
\hline$C F_{2} O$ & 682 & 795 & NA \\
\hline $\mathrm{CHF}_{3}$ & 906 & 757 & $\mathrm{NA}$ \\
\hline $\mathrm{CH}_{2} \mathrm{~F}_{2}$ & 953 & 786 & NA \\
\hline$H C L$ & 332 & 570 & 643 \\
\hline$C C L_{2} \mathrm{O}$ & 1661 & 1450 & NA \\
\hline
\end{tabular}

Table 5: The BKWC parameters and covolumes.

We will now evaluate the results obtained with the BKWCEOS. In the following series of figures we graph theoretical values versus experimental values for a range of parameters. We also give RMS errors, as opposed to RMS discrepancies, since BKWC was specifically designed to minimize 
the RMS discrepancy. This yields a fairer comparison between the various parametrizations. In Figure 1 we show the detonation velocity for BKWC, BKWR, and BKWS. The BKWS calculations were done with the reduced product set described above; nearly identical results were obtained in test cases with the full product set. For this set of compounds, BKWC has the best match with experiment, showing a $0.8 \%$ RMS error. The biggest difference between the parameter sets occurs when $D$ is low (under $6 \mathrm{~mm} / \mu \mathrm{s}$ ). BKWR overestimates $D$ in this case, while BKWS tends to underestimate $D$. BKWC shows very good agreement with experiment in the low $D$ limit.

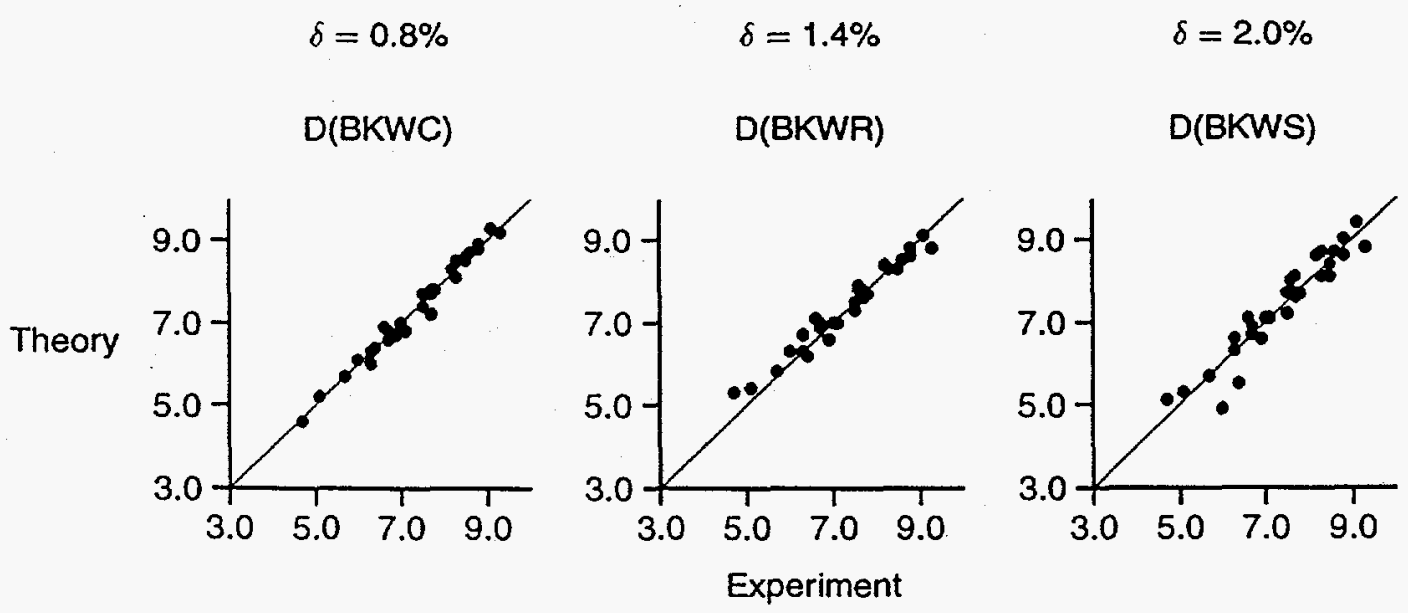

Figure 1: Theoretical versus experimental detonation velocities for the BKW EOS in $m m / \mu s$.

Larger disagreement with experiment is found for the C-J pressure. It is important to remember, however, that the $\mathrm{C}-\mathrm{J}$ pressure has much higher experimental error bars associated with it than the detonation velocity. C-J pressure measurements are usually indirect extrapolations. In the present case, $P_{C J}$ was determined from a JWL[25] fit to cylinder test results, with the exception of PETN, which was determined by supracompression experiments[26].

It is thus difficult to ascertain how much of the increased disagreement is due to experimental error, and how much is due to the EOS. Figure 2 compares experimental and theoretical C-J pressures. Here the BKWR equation of state fairs slightly better than BKWC. BKWC and BKWS predict values of $P_{C J}$ that are lower than the experimental ones. It is interesting to note that most equilibrium codes (e.g. TIGER[2], CHEETAH[1],CHEQ[3]) predict C-J pressures which are systematically lower than measured values. The reason for this is unknown.

One of the most significant improvements made with BKWC is that the shape of the adiabat is reproduced. In Figure 3 the adiabat energy at a relative volume of 2.2 is displayed. BKWC is a dramatic improvement over BKWR, and is also significantly better than BKWS. The BKWR equation of state systematically underestimates (i.e. gives too negative a number) the adiabat energy. In the past this was fixed empirically by using scale factors determined to match PETN cylinder test results[9]. Scale factors were not used in the reported calculations. BKWS adiabat energies also were systematically too low for high performance explosives, and too high for low performance explosives. BKWC predicts somewhat low values for low and high performance materials, and shows no bias for intermediate performance $(E=-6$ to $-4 \mathrm{~kJ} / \mathrm{cc})$. 
$\delta=3.4 \%$

$P_{C J}(\mathrm{BKWC})$

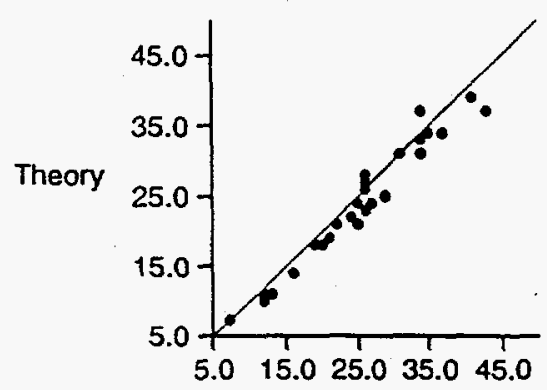

$\delta=3.0 \%$

$P_{C J}$ (BKWR)

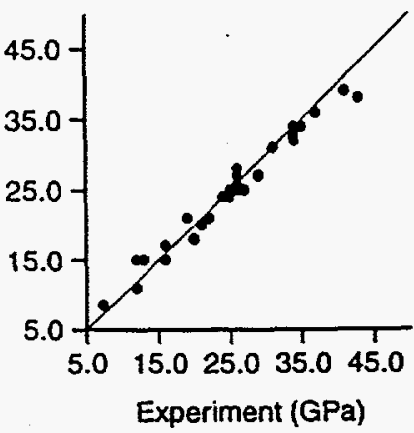

xperiment (GPa) $\delta=4.3 \%$

$P_{C J}$ (BKWS)

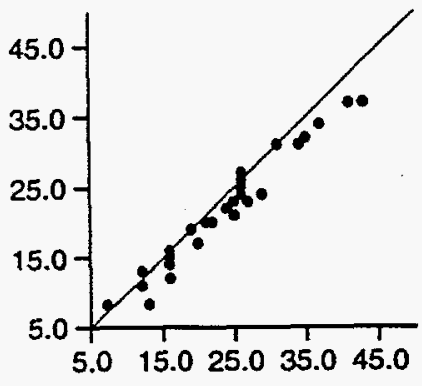

Figure 2: Experimental and theoretical C-J pressures for the BKW EOS.
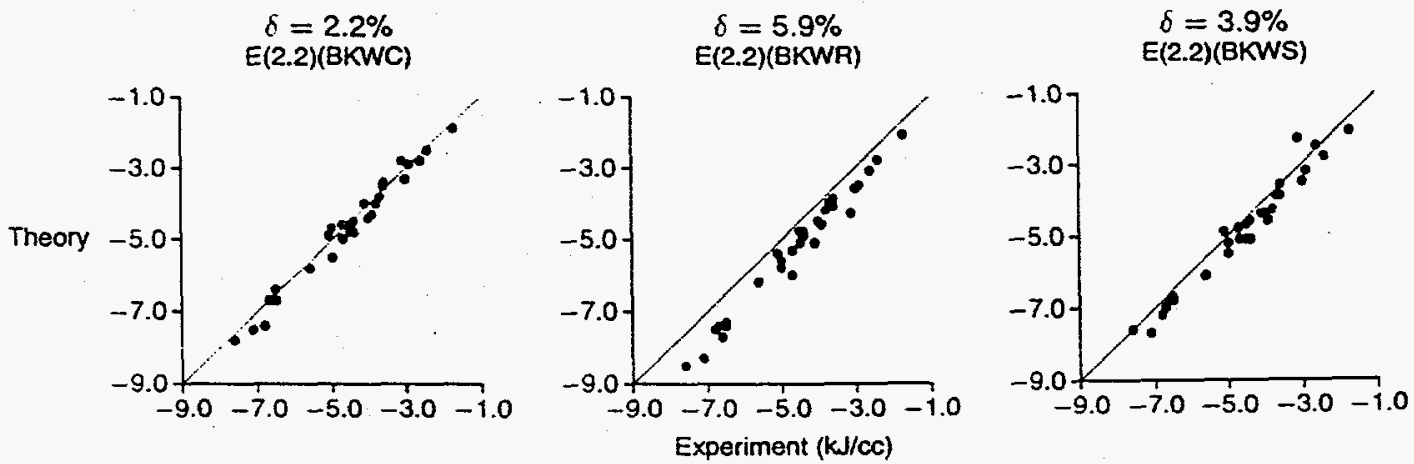

Figure 3: Experimental and theoretical adiabat energies at a relative volume of 2.2 for the BKW EOS.
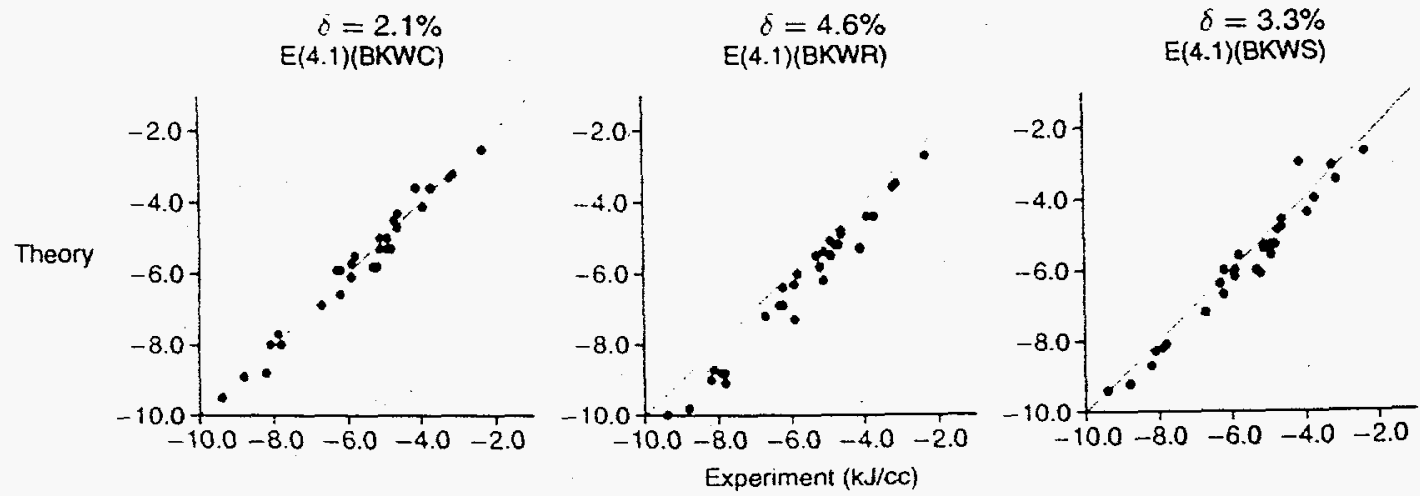

Figure 4: Experimental and theoretical adiabat energies at a relative volume of 4.1 for the BKW EOS. 


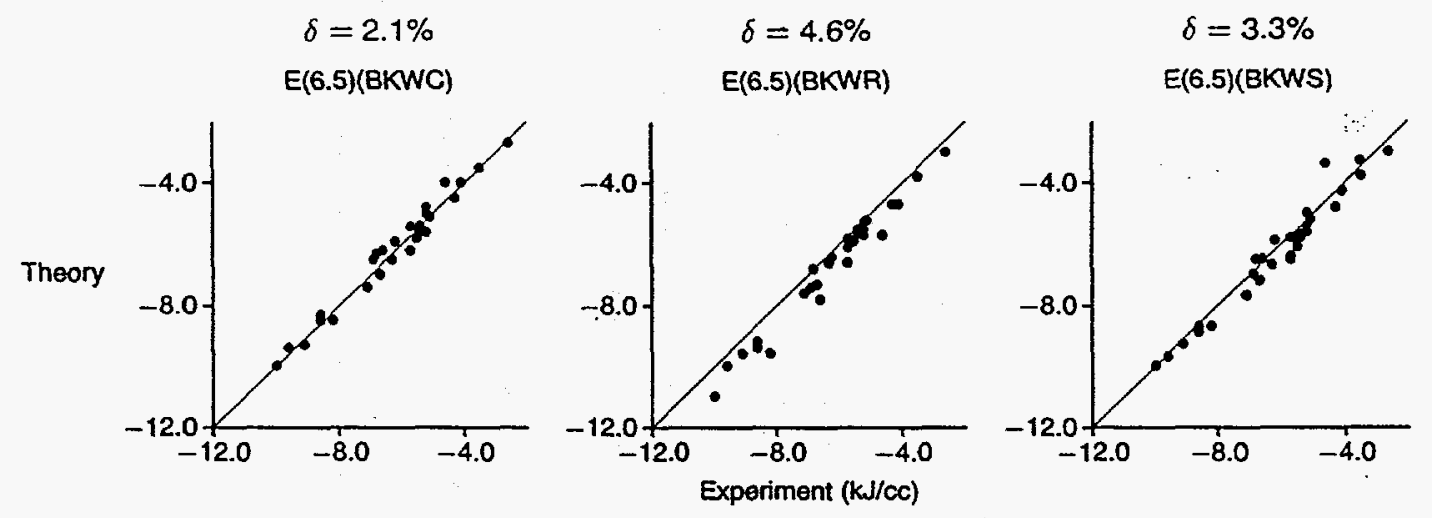

Figure 5: Experimental and theoretical adiabat energies at a relative volume of 6.5 for the BKW EOS.

The adiabat at larger volumes is also well reproduced. In Figures 4 and 5 we show the adiabat energy at a relative volume of 4.1 and 6.5. BKWC again shows the smallest errors. BKWR tends to be too low, by roughly the same amount as when $v=2.2$. BKWS shows the greatest scatter for low performing explosives with $E$ between -4 and $-2 \mathrm{~kJ} / \mathrm{cc}$. For $v=4.1$ It tends to be low in the range -8.0 to $-5.0 \mathrm{~kJ} / \mathrm{cc}$, and high in the range -5.0 to $-2.0 \mathrm{~kJ} / \mathrm{cc}$. Similar trends are seen when $v=6.5$.

As a final comparison, we plot the mechanical energy of detonation in Figure 6 . We define the mechanical energy of detonation to be the energy difference between the reactants and the products at the end of the adiabat. The end of the adiabat is taken to be 1 ATM or $298 \mathrm{~K}$, whichever is encountered first along the expansion. The temperature condition is necessary because thermochemical reference data used in CHEETAH is not reliable at low temperatures. The mechanical energy of detonation is thus a measure of the total PV work an explosive is capable of delivering. $\mathrm{BKWC}$ performs well in predicting the mechanical energy of detonation, with an RMS error of 2.3 $\%$. BKWR is the worst, with a $3.3 \%$ RMS error.

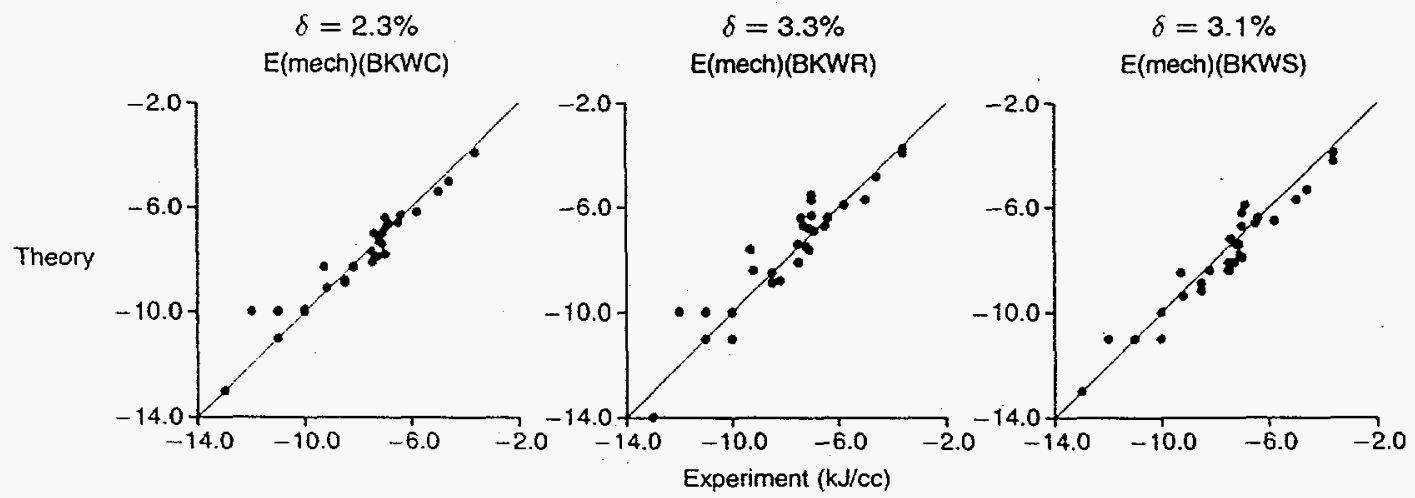

Figure 6: Experimental and theoretical mechanical energies of detonation for the BKW EOS. 
In the results so far, we have compared the performance of the BKWC parameter set to other $\mathrm{BKW}$ parameter sets in predicting the performance database. In judging this comparison, it is important to remember that BKWC was specifically fit to the performance database. It is possible that the parameter set would fair less well when applied to materials outside the database. It is a difficult task to find experimental data for properties that are truly independent of the performance database. For instance, the majority of cylinder tests performed at LLNL were formulated HMX and TATB - the materials of greatest interest to the Department of Energy. Comparatively few experiments were done on other materials.

Nonetheless, it is possible to find detonation velocities for a wide range of materials. We have collected a detonation velocity database that covers many materials not included in the cylinder test performance database. Detonation velocities were taken from several reference works: the LLNL explosives handbook[27], Meyer's handbook[28], the recent review article by Anderson[29], and recent experiments on TNAZ[30]. In all, the detonation velocity database contained 127 entries. In Table 7, we give RMS errors for the detonation velocities, sorted by reference.

BKWC is the best parameter set for reproducing the detonation velocity database. It has the lowest RMS errors for every reference except the recent TNAZ measurements. The TNAZ data, however, only reflects two experiments whereas the other references have a much larger number of experiments. There is an interesting variation in the RMS error with reference. References covering well-studied explosives (LLNL, Anderson) are better reproduced by BKWC than references covering more exotic explosives (Meyer). This is probably due to the quality of the experimental data: large scale, accurate experiments are rarely done on materials of little practical interest. On the other hand, an alternative explanation could hold: the rarer explosives have unusual properties that are not well reproduced by the BKW equation of state. This question can only be resolved through careful, size-converged experiments on unusual materials.

\subsection{Conclusion}

Our goal in performing the calculations described here was to obtain a BKW parametrization that worked well for the whole detonation process when applied to a wide range of compounds. For the properties that we have considered, the BKWC EOS yields results that are usually within estimated error bars of the experimental results.

Another interesting feature of our work is the use of an empirical carbon model. A parallel effort at LLNL on the CHEQ thermochemical code produced a non-equilibrium carbon model with a higher density than standard carbon[3,21]. We find this to be a puzzling result. Intuitively one would expect non-equilibrium carbon to resemble amorphous carbon, and thus have a lower density than crystalline carbon. It may very well be that the empirical carbon model is compensating for other EOS errors, and therefore the values of the parameters themselves have no independent physical meaning.

We would also like to caution researchers about using the BKWC EOS for properties other than the ones considered here. For instance, thermochemical codes predict chemical concentrations along the adiabat. We have no idea how well such properties will be predicted by BKWC (or any other equation of state. for that matter) since there is no reliable experimental data to compare to. Within the group of properties considered here, however, BKWC does well and will probably be a useful tool for fast thermochemical calculations. 


Compound
Ammonium picrate
Ammonium nitrate (AN)
BTF
Comp B-3
Comp B
Comp C-3
Comp C-4
Cyclotol 75
Cyclotol 60
Cyclotrimethylenenitrosamine
Cyanuric triazide
DATB
DEGN
DINA
Dinitrodimethyloxamide
Diazodinitrophenol
Diethyleneglycol dinitrate
Dinitrodimethyloxamide
Dinitrophenoxyethyinitrate
DIPAM
DIPEHN
DNB
EDD
EDNA
Ethyl picrate
Ethriol trinitrate
Ethyl nitrate
FEFO
HMX
HNAB
HNDP
HNS
Hydrazine nitrate
LX01
LX04
LX07
LX09
LX10-0
LX10-1
LXI1

$\begin{array}{ll}\text { No. } & \text { Ref. } \\ 3 & {[29,27,28]} \\ 2 & {[27]} \\ 1 & {[27]} \\ 1 & {[27]} \\ 4 & {[27]} \\ 1 & {[27]} \\ 1 & {[27]} \\ 2 & {[27]} \\ 1 & {[27]} \\ 1 & {[28]} \\ 1 & {[28]} \\ 3 & {[29,27]} \\ 2 & {[27,28]} \\ 1 & {[28]} \\ 1 & {[28]} \\ 1 & {[28]} \\ 1 & {[28]} \\ 1 & {[28]} \\ 1 & {[28]} \\ 1 & {[28]} \\ 1 & {[28]} \\ 1 & {[28]} \\ 1 & {[28]} \\ 1 & {[28]} \\ 1 & {[28]} \\ 1 & {[28]} \\ 1 & {[28]} \\ 1 & {[27]} \\ 2 & {[27,28]} \\ 1 & {[27]} \\ 1 & {[28]} \\ 3 & {[29,27]} \\ 1 & {[28]} \\ 1 & {[27]} \\ 1 & {[27]} \\ 1 & {[27]} \\ 1 & {[27]} \\ 1 & {[27]} \\ 1 & {[27]} \\ 1 & {[27]} \\ & \end{array}$

$\begin{array}{ll}\text { Compound } & \text { No } \\ \text { LX14 } & 1 \\ \text { LX14 } & 1 \\ \text { LX17 } & 1 \\ \text { MEN-2 } & 1 \\ \text { Methyl nitrate } & 1 \\ \text { MHN } & 1 \\ \text { Nitropentanon } & 1 \\ \text { Nitroglycerine } & 3 \\ \text { Nitroglycol } & 1 \\ \text { NM } & 2 \\ \text { NQ } & 2 \\ \text { NTO } & 2 \\ \text { Octol 75 } & 2 \\ \text { Octol 70 } & 1 \\ \text { PBX9007 } & 1 \\ \text { PBX9010 } & 1 \\ \text { PBX9011 } & 1 \\ \text { PBX9205 } & 1 \\ \text { PBX9407 } & 1 \\ \text { PBX9501 } & 1 \\ \text { PBX9503 } & 1 \\ \text { Pentastit } & 1 \\ \text { Pentolite } & 3 \\ \text { PETN } & 2 \\ \text { Picryl chloride } & 1 \\ \text { Picric acid } & 3 \\ \text { RDX } & 4 \\ \text { Tacot } & 2 \\ \text { TAGN } & 1 \\ \text { TATB } & 3 \\ \text { Tetryl } & 5 \\ \text { TNA } & 1 \\ \text { TNAN } & 1 \\ \text { TNAZ } & 2 \\ \text { TNB } & 1 \\ \text { TNC } & 1 \\ \text { TNGU } & 1 \\ \text { TNM } & 2 \\ \text { TNP } & 1 \\ \text { TNT } & 6 \\ \text { TNPEN } & 1\end{array}$

Ref.

[27]

[27]

[27]

[27]

[28]

[28]

[28]

$[27,28]$

[28]

[27, 28]

$[29,27]$

[29]

$[27,28]$

[28]

[27]

[27]

[27]

[27]

[27]

[27]

[27]

[28]

$[27,28]$

[27]

[28]

$[27,28]$

$[29,28,27]$

$[27,28]$

[28]

$[29,27,28]$

$[29,27,28]$

[28]

[28]

[30]

[28]

[28]

[28]

$[27,28]$

[28]

$[27,28]$

[28]

Table 6: Compounds contained in the detonation velocity database. No. is the number of entries for the particular compound.

$\begin{array}{lllll}\text { Reference } & \text { No. entries } & \text { BKWC } & \text { BKWR } & \text { BKWS } \\ \text { LLNL } & 68 & 2.57 & 3.72 & 4.22 \\ \text { TNAZ } & 2 & 2.48 & 1.15 & 1.52 \\ \text { Anderson } & 10 & 2.03 & 2.34 & 3.12 \\ \text { Meyer } & 47 & 4.01 & 5.59 & 6.71\end{array}$

Table 7: RMS percent errors in predicting detonation velocites 


\section{Acknowledgements}

CHEETAH owes an obvious debt to $\mathrm{M}$. Cowperwaithe and W. H. Zwisler, the developers of TIGER [2]. Many of the capabilities that will be put into CHEETAH are inspired by the thermochemical code CHEQ, written by A. Nichols III and F. Ree. P. Clark Souers first advanced the idea of improving TIGER, and has been instrumental in testing the program. Clark has also assembled the reactant library described in the User's Manual[1]. Randall Simpson and Al Holt have been relentless proponents of the project. Milt Finger provided initial funding. Ed James and Mark Hoffman were the first brave users of the PC version. Mike Murphy loaned me computer hardware necessary to develop CHEETAH for the PC and helped with numerous file transfers. Craig Tarver has made several insightful suggestions. Finally, I have had many useful conversations with Albert Nichols III; his help has been invaluable. The cylinder test data reported here were generated over a thirty year period by Howard Hornig, Jim McDonnell, Don Ornellas, Frank Helm, Don Breithaupt, and Milt Finger.

\section{References}

[1] L. E. Fried, CHEETAH 1.0 User's Manual, UCRL-MA-117541, University of California, 1994.

[2] M. Cowperthwaite and W. H. Zwisler, "TIGER Computer Program Documentation", SRI Publication No. Z106, 1973.

[3] A. L. Nichols III and F. H. Ree, CHEQ 2.0 User's Manual, UCRL-MA-106754, University of California, 1990.

[4] W. Fickett and W. C. Davis. Detonation, University of California Press, Berkeley, CA, 1979.

[5] G. B. Kistiakowsky and E. B. Wilson. "Report on the prediction of detonation velocities of solid explosives". Office of Scientific Research and Development report OSRD-69 (1941); R. D. Cowan and W. Fickett. J. Chem. Phys., 24, 932(1956).

[6] C. L. Mader, "Numerical Modeling of Detonations", University of California Press, Berkeley, 1979.

[7] M. Finger, E. Lee, F.H. Helm, B. Hayes, H. Hornig, R. McGuire, M. Kahara, and M. Guidry, "The Effect of Elemental Composition on the Detonation Behavior of Explosives", Proceedings of the Sixth Symposium (International) on Detonation, Coronado, CA, 24-27 August 1976. Office of Naval Research. Arlington. VA, ACR-221, p. 710-722.

[8] M.L. Hobbs and M.R. Baer. "Nonideal Thermoequilibrium Calculations Using a Large Prod"ct Species Data Base". Sandia Report SAND92-0482(1992), Sandia National Laboratories, Albuquerque, NM. 87185: M.L. Hobbs and M.R. Baer, "Calibrating the BKW-EOS with a Large Product Species Data Base and Measured C-J Properties", Tenth Symposium (International) on Detonation, Boston, MA (1994). 
[9] P.C. Souers and J.W. Kury, "Comparison of cylinder data and code calculations for homogeneous explosives, Propellants, Explosives, Pyrotechnics, 18, 175(1993).

[10] M. Cowperthwaithe and W. H. Zwisler, "The JCZ equations of state for detonation products and their incorporation into the TIGER code", Sixth Detonation Symposium, p. 162, (1976).

[11] A.L. Nichols III, private communication, LLNL, 1993.

[12] P. C. Souers and L. C. Haselman, Jr. Detonation Equation of State at LLNL, 1993, UCRL-ID116113, University of California, 1994.

[13] Lawrence Livermore National Laboratory, "High Explosive Applications Laboratory Records, 1976-1991". A loose-leaf Cylinder Handbook contains much of the data.

[14] "LASL Explosive Property Data", T.R. Gibbs and A. Popolato (eds.), University of California Press, Berkeley, 1980.

[15] P.E. Kramer, “HNS Cylinder Tests”, Report MHSMP-75-17 (1975), Mason and Hanger-Silas Mason Co., Pantex Plant, Amarillo, TX, USA.

[16] P. C. Souers, D. B. Larson, and C. M. Tarver, "Performance calculations on the ANFO explosive RX-HD”, UCRL-ID-1 16113, University of California, 1994.

[17] D. L. Ornellas, "Calorimetric determinations of the heat and products of detonation for explosives: October 1961 to April 1982", Report UCRL-52821 (1982), Lawrence Livermore National Laboratory, Livermore, CA, USA.

[18] "JANAF Thermochemical Tables", 3rd ed., Journal of Physical and Chemical Reference Data, Vol. 14, 1985, Supplement No. 1, American Institute of Physics, New York, 1986.

[19] A. W. Campbell, Propellants, Explosives, and Pyrotechnics, 9, 183(1984).

[20] F.H. Ree, J. Chem. Phys., 81, 1251(1984); J. Chem. Phys., 84, 5845(1986).

[21] M. VanThiel, private communication, 1994, Lawrence Livermore National Laboratory, Livermore, CA, USA.

[22] M. van Thiel and F.H. Ree, J. Appl. Phys., 62, 1761-1767(1987).

[23] G. Dueck, "New Optimization Heuristics: The Great Deluge Algorithm and the Record-toRecord Travel", J. Comp. Phys., 104, 8-92(1993).

[24] S. Kirkpatrick, C.D. Gelatt, Jr., and M.P. Vecchi Optimization by simulated annealing, Science, 220 671(1983); S. Kirkpatrick, Optimization by simulated annealing: quantitative studies, J. Stat. Phys., 34, 975(1984).

[25] J.W. Kury, H.C. Hornig, E.L. Lee, J.L. McDonnel, D.L. Ornellas, M. Finger, F.M. Strange, and M.L. Wilkins, "Metal Acceleration by Chemical Explosives", Proceedings of the Fourth Symposium (International) on Detonation, White Oak, MD 12-15 October 1965, p. 3-12. 
[26] L. Green, N. Holmes, and J. Kury, "Shock Measurements on Explosives in the SupraCompressive Region", International Symposium on Pyrotechnics and Explosives, Beijing, China, 12-15 October 1987; Report UCRL-95461 (1987), Lawrence Livermore National Laboratory, Livermore, CA, USA.

[27] Dobratz, B.M. and Crawford, P.C. LLNL Explosives Handbook, UCRL-52997, 1985.

[28] R. Meyer, Explosives, VCH, Weinheim, 1987.

[29] E. Anderson, Explosives, in Tactical Missile Warheads, J. Carleone, ed., Vol. 155 of Progress in Astronautics and Aeronautics, American Institute of Aeronautics and Astronautics, Washington, DC, ISBN 1-56347-067-5.

[30] S. A. Aubert, WL/MNME, private communication, 1994. 